\title{
Pharmacokinetic comparison of soy isoflavone extracts in human plasma
}

\section{Authors and affiliations}

Jose Rodríguez-Moratón ${ }^{1,2,3, \#}$, Magí Farré ${ }^{1,4, \#}$, Clara Pérez-Mañãa ${ }^{1,4}$, Esther Papaseit ${ }^{1,4}$, Roser Martínez-Riera ${ }^{4,5}$, Rafael de la Torre ${ }^{1,2,3^{*}}$, Nieves Pizarro ${ }^{1,4}$

1 Integrative Pharmacology and Systems Neuroscience Research Group, Neurosciences Research Program, IMIM (Hospital del Mar Medical Research Institute), Dr. Aiguader 88, Barcelona 08003, Spain.

${ }^{2}$ Pompeu Fabra University (CEXS-UPF), Dr. Aiguader 88, Barcelona 08003, Spain

${ }^{3}$ CIBER de Fisiopatología Obesidad y Nutrición, Santiago de Compostela 15706, Spain.

${ }^{4}$ Universitat Autònoma de Barcelona (Department of Pharmacology, Therapeutics and Toxicology; Department of Psychiatry), UAB Campus, M Building, Barcelona 08193, Spain.

${ }^{5}$ Institut de Neuropsiquiatria i Adiccions. Adiction Unit and IMIM, Dr. Aiguader 88, Barcelona 08003, Spain.

\# Authorship credit should be equally distributed among the authors independently of the order.

*Corresponding author: Rafael de la Torre, PharmD, PhD. Tel: +34 933160484; Fax: +34 933160467; E-mail: rtorre@imim.es. 
2 The soy isoflavones daidzein and genistein produce several biological activities 3 related to health benefits. A number of isoflavone extracts are commercially 4 available, but there is little information concerning the specific isoflavone content of 5 these products or differences in their bioavailability and pharmacokinetics. This 6 study describes the development and validation of an analytical method to detect 7 and quantify daidzein, genistein, and equol in human plasma using liquid 8 chromatography coupled to tandem mass spectrometry (LC/MS/MS). The method 9 was applied in a crossover, randomized, bioavailability study. Twelve healthy 10 volunteers were administered the same total isoflavones dose from two isoflavone 11 supplement preparations (Super-Absorbable Soy Isoflavones ${ }^{\circledR}$ (Life Extension, US) 12 and Fitoladius ${ }^{\circledR}\left(\right.$ Merck, Spain)). The pharmacokinetic parameters $\left(\mathrm{AUC}_{0-24} /\right.$ Dose $^{2}$ 13 and $\mathrm{C}_{\max } /$ Dose) of the isoflavones from the two preparations differed significantly. 14 Such differences in bioavailability and kinetics may have relevant effects on the 15 health benefits derived from their intake.

\section{$17 \quad$ Keywords}

18 Soy isoflavone extracts, daidzein, genistein, equol, bioavailability, 19 pharmacokinetics, clinical trial. 


\section{INTRODUCTION}

Many isoflavones act as phytoestrogens due to de fact that these compounds present similarities in chemical structure and properties to endogenous steroids (e.g. 17- $\beta$ estradiol). The most important dietary sources of isoflavones are soy beans and soy foods. The soy isoflavones aglycones daidzein (4',7-dihydroxyisoflavone), equol (4',7dihydroxy-3,4-dihydroisoflavone), and genistein (4',5,7-trihydroxyisoflavone) (See Figure 1) display a number of biological activities which have been related to lower occurrences of cardiovascular diseases, osteoporosis, hormone-dependent cancers, adverse menopausal manifestations, and age-related cognitive decline. ${ }^{1,2}$

In order to evaluate the importance of isoflavones on human health, it is necessary to have a better understanding of the bioavailability and pharmacokinetics of these compounds. Isoflavone disposition follows several deconjugation and conjugation steps. Soy and unfermented soy food isoflavones are mainly found as glycosides (e.g. daidzin, genistin, and glycitin), they display high hydrophilicity and molecular weights, and are the object of a number of metabolic biotransformations. Firstly, gut bacterial $\beta$ glucosidases ${ }^{3}$ and the gastric acidic $\mathrm{p} H$ carry out the hydrolysis of the sugar moiety. This facilitates the absorption process ${ }^{4,5}$ that takes place by passive diffusion in the upper small intestine. ${ }^{6}$ Then, the intestinal ${ }^{7}$ and hepatic ${ }^{8}$ phase II enzymes UDPglucuronyl transferases and PAPS-sulfotransferases carry out the corresponding conjugation with glucuronic acid and sulphate. The excretion of these conjugates can be both renal and biliary, the later undergoing enterohepatic recirculation, which leads to deconjugation, reabsorption, and further metabolism. ${ }^{1,5}$ Most circulating isoflavones are in the form of glucuronides ${ }^{9}$ and a small fraction is sulphated or left unconjugated. ${ }^{1}$ Although it is generally believed that aglycones are the most active biological compounds in humans, conjugates have also been postulated as displaying biological 
46 activities $^{10}$ and some of them, such as sulphates, have been reported to have higher 47 activities than the corresponding aglycones. ${ }^{11}$

48 Whereas daidzein and genistein are present in soy foods, equol is a metabolite of daidzein whose production depends upon the individual gut microflora ${ }^{12}$ and only 30 $50 \%$ of the adult Caucasian population is able to produce it. ${ }^{1}$ Unlike daidzein or 51 genistein, equol presents a stereogenic centre at C-3, and therefore different 52 enantiomeric forms exist. In humans, $(S)$-equol is the only enantiomer which is 53 produced by intestinal bacterial flora. ${ }^{13}$ This fact is of clinical relevance since both 54 enantiomers have different activities; $(S)$-equol, but no $(R)$-equol, displays high affinity 55 for the oestrogen receptor beta $(\mathrm{ER} \beta)$ and its activity is even higher than that of estradiol. $^{13}$

57 Because of the beneficial health effects that soy isoflavones may provide to humans, 58 several brands of isoflavone extracts have been commercialized over the last decades.

59 However, there is little information concerning these preparations with respect to their 60 specific isoflavone profile and quantity. Differences in bioavailability and 61 pharmacokinetics may have an impact on isoflavone biological and pharmacological 62 activities.

63 A number of analytical methods have been developed to detect and quantify 64 isoflavone concentrations in biological fluids (plasma, serum, and urine). The most 65 commonly used include gas chromatography coupled to mass spectrometry (GC/MS), ${ }^{14}$ 66 high performance liquid chromatography (HPLC) with electrochemical detection, ${ }^{15}$ 67 HPLC with UV detection, ${ }^{16}$ and liquid chromatography coupled to mass spectrometry 68 (LC/MS). ${ }^{4}{ }^{17}$ At present, LC/MS/MS is the most commonly employed analytical 69 technique due to its high sensitivity and selectivity. 
The aim of the present study was to develop and validate an LC/MS/MS method

71 for bioavailability studies of commercial isoflavone preparations which would be

72 subsequently used in clinical research. A randomized, crossover, bioavailability study of

two different, commercially available soy isoflavone extracts (Soy Isoflavones ${ }^{\circledR}$ (Life

Extension, US) and Fitoladius ${ }^{\circledR}$ (Merck, Spain)) was performed.

\section{MATERIALS AND METHODS}

Chemicals and reagents

Daidzein, $o$-phosphoric acid (85\%), $\beta$-Glucuronidase from Helix pomatia (H-2 and H-1), $\beta$-Glucuronidase from Escherichia coli, citric acid monohydrate and sodium 79 citrate dihydrate were purchased from Sigma-Aldrich Corporation (St. Louis, MO, USA). Equol and genistin were purchased from LC Laboratories (Woburn, MA, USA).

81 Genistein was purchased from Extrasynthèse (Lyon, France). Daidzein-4-sulphate 82 disodium salt, daidzein-7- $\beta$-D-glucuronide potassium salt, daidzein-7- $\beta$-D-glucuronide-

83 4-sulphate disodium salt and equol-4-sulphate were custom synthesized by Toronto 84 Research Chemicals Inc. (Toronto, Ontario, Canada). Daidzein- $\mathrm{d}_{6}$ and equol- $\mathrm{d}_{4}$ were 85 purchased from Medical Isotopes Inc. (Pelham, NH, USA). Methanol, acetonitrile 86 (HPLC gradient grade), ammonium hydroxide and acetic acid were purchased from

87 Merck (Darmstadt, Germany). Ultrapure water was obtained using a Milli-Q 88 purification system (Millipore Ibérica, Barcelona, Spain). Drug-free plasma was 89 obtained from the Hospital del Mar blood bank (Barcelona, Spain).

\section{$90 \quad$ Working standards}

91 Standard stock solutions of all analytes and their corresponding internal 92 standards (IS), with the exception of daidzein-7- $\beta$-D-glucuronide and daidzein-7- $\beta$-D93 glucuronide-4-sulphate where water was used were prepared by dissolving $10 \mathrm{mg}$ of 
94 each substance in $10 \mathrm{~mL}$ of methanol. Working solutions were prepared by further

95 diluting the starting solutions and were kept in dark flasks at $-20^{\circ} \mathrm{C}$.

96

\section{Instrumentation: LC/MS/MS conditions}

Identification and quantification analysis was performed using an Agilent 1200 series HPLC system (Agilent technologies) coupled to a triple quadrupole (6410 Triple Quad LC/MS; Agilent) mass spectrometer with an electrospray interface. Nitrogen was employed as a drying and nebulizing gas. A capillary voltage of $4 \mathrm{kV}$ in negative ionization mode was used and the source temperature was set to $325^{\circ} \mathrm{C}$.

The liquid chromatographic separation of daidzein, equol and genistein was performed at $40{ }^{\circ} \mathrm{C}$ on an Acquity UPLC ${ }^{\circledR}$ BEH $\mathrm{C}_{18}$ column $(100 \mathrm{~mm}$ x $3.0 \mathrm{~mm}$ i.d., 1.7 $\mu \mathrm{m}$ particle size) (Waters Corporation). The mobile phase was an isocratic solvent system consisting of $0.01 \%$ ammonium acetate at $\mathrm{pH} 5$ and methanol (40:60, v/v) at a flow rate of $0.3 \mathrm{~mL} / \mathrm{min}$. Injection volume was $10 \mu \mathrm{L}$. The total run time was $5 \mathrm{~min}$ per sample. All compounds were monitored in negative ionization using the multiple reaction mode. Daidzein and genistein were quantified by comparing their peak area ratios with daidzein- $\mathrm{d}_{6}$ (IS1) whereas equol was quantified using equol- $\mathrm{d}_{4}$ as internal standard (IS2). Due to the chemical structure similarities between daidzein and genistein, daidzein- $\mathrm{d}_{6}$ was used as the internal standard of genistein.

\section{Sample preparation}

Firstly, $1 \mathrm{~mL}$ aliquot of plasma sample was transferred into a glass tube and spiked with $30 \mu \mathrm{L}$ of IS1 solution (containing daidzein- $\mathrm{d}_{6} 10 \mu \mathrm{g} / \mathrm{mL}$ ) and $10 \mu \mathrm{L}$ of IS2 (containing $10 \mu \mathrm{g} / \mathrm{mL}$ of equol- $\mathrm{d}_{4}$ ). Approximately 3000 units of $\beta$-glucuronidase $\mathrm{H} 1$ (2 $\mathrm{mg} / \mathrm{mL}$, in $0.1 \mathrm{M}$ sodium citrate buffer, $\mathrm{pH}=5$ ) were added and sample was incubated at $37^{\circ} \mathrm{C}$ for 16 hours in a shaking water bath. Hydrolysis time could be reduced to 1 hour if the temperature of the water bath was increased to $55^{\circ} \mathrm{C}$. 
After cooling to room temperature, $1 \mathrm{~mL}$ of $4 \% \mathrm{H}_{3} \mathrm{PO}_{4}$ was added and all the

120 tubes were centrifuged at $3500 \mathrm{rpm}$ for 5 minutes. They were then submitted to a solid-

121 phase extraction (SPE) procedure using Oasis ${ }^{\circledR}$ MAX cartridges (3cc, 60-mg; Waters

122 Corporation, Dublin, Ireland). Cartridges were conditioned with $2 \mathrm{~mL}$ of methanol and

123 equilibrated with $2 \mathrm{~mL}$ of water. After sample loading, interferences were washed away

124 with $2 \mathrm{~mL}$ of $5 \% \mathrm{NH}_{4} \mathrm{OH}$ and $2 \mathrm{~mL}$ of $2 \% \mathrm{HCOOH}$ containing $10 \%$ methanol. The

125 compounds of interest were then eluted with $2 \mathrm{~mL}$ of a solution of methanol containing

$1262 \% \mathrm{HCOOH}$. After the evaporation of the solvent $\left(<29^{\circ} \mathrm{C},<10 \mathrm{psi}\right)$, analytes were

127 reconstituted in $100 \mu \mathrm{L}$ of $\mathrm{CH}_{3} \mathrm{COONH}_{4} 0.01 \%$, $\mathrm{pH}=5 / \mathrm{MeOH}(50 / 50)$. Finally, samples

128 were centrifuged at $12,000 \mathrm{rpm}$ for $10 \mathrm{~min}$ at $4{ }^{\circ} \mathrm{C}$ and supernatants analyzed by $129 \mathrm{HPLC} / \mathrm{MS} / \mathrm{MS}$.

\section{$130 \quad$ Method validation}

\section{Calibration curves and linearity}

Calibration standards of daidzein, equol, and genistein were prepared each analysis day and consisted of two replicates at 6 different concentrations $(5,50,200$, 500,750 , and $1000 \mathrm{ng} / \mathrm{mL}$ ). Calibration curves were prepared by adding the appropriate volume of working solutions, evaporating the solvent under $\mathrm{N}_{2}\left(25^{\circ} \mathrm{C}, 10 \mathrm{psi}\right)$, and adding $1 \mathrm{~mL}$ of isoflavone-free plasma. Calibration curves with their corresponding

137 slope (s), intercept and correlation coefficient (r) were calculated by weighting $(1 / \mathrm{x})$

138 least-squares linear regression of the peak area ratio (analyte/IS) versus the 139 concentration of the standards. The method was considered linear if the coefficient of 140 determination was greater than 0.99 .

\section{Intra-assay accuracy and precision}

142 In order to evaluate the precision and accuracy of the method, quality control

143 (QC) samples were prepared by spiking known amounts of the analytes to isoflavone- 
144 free human plasma in order to achieve final concentrations of 5 (lower limit of

145 quantification; LLOQ), 10 (low QC), 100 (medium QC), and 800 (high QC) ng/mL of

146 daidzein, equol, and genistein. Five replicates of each QC were analysed against the

147 calibration curve, and the obtained concentrations were compared to their nominal 148 value.

149 Intra-assay accuracy was expressed as the \% relative error of the estimated 150 concentrations. It was evaluated by five replicates of the QC samples that were analysed 151 in one day, at each concentration level. Accuracy was acceptable if the mean 152 concentration was within $15 \%$ of the nominal values with the exception of the LLOQ 153 which was acceptable if it was within $20 \%$ of the nominal value.

154 Intra-assay precision of the method was calculated as the relative standard 155 deviation (RSD, \%) of the estimated concentrations obtained for five replicates of the 156 QC samples that were analysed in one day, at each concentration level. Precision was 157 acceptable if the RSD was within $15 \%$ of the nominal values with the exception of the 158 LLOQ which was acceptable if it was within $20 \%$ of the nominal value.

\section{Inter-assay accuracy and precision} Inter-assay accuracy and inter-assay precision were calculated as described for

161 intra-assay accuracy and precision with data obtained during three validation assays that 162 took place during three different days.

\section{Limit of detection (LOD)}

164 The LOD was calculated using the standard deviations (SD) of the obtained 165 concentrations from five replicates of the LLOQ $(5 \mathrm{ng} / \mathrm{mL})$ and the slope (s) of the 166 calibration curve, according to the following formula: LOD $=3.3 *(\mathrm{SD} / \mathrm{s})$.

167 Additionally, at the estimated LOD of the surrogated analytes, it was confirmed that the 168 LOD was at least 3 times the signal to noise ratio of a blank plasma sample. 


\section{Lower limit of quantification (LLOQ)}

The LLOQ was the lowest concentration calibrator experimentally tested that presented acceptable accuracy $(80-120 \%)$ and precision $( \pm 20 \%)$.

173 The extraction process recovery was evaluated by comparing the responses of a

174 mixture of standards with and without extraction. For this purpose, six different sources

175 of isoflavone-free plasma were spiked at a low $(5 \mathrm{ng} / \mathrm{mL})$ and high $(800 \mathrm{ng} / \mathrm{mL})$ level of

176 concentration. The extraction recovery was calculated as the quotient of response of

177 standard solutions spiked into plasma before extraction (with standard) and the response

178 of standard solutions spiked into plasma after extraction. Results are expressed as a 179 percentage.

\section{Matrix effect}

In a similar way, the matrix effect was analysed using six different batches of

182 isoflavone-free plasma at two concentrations (5 and $800 \mathrm{ng} / \mathrm{mL}$ ). It was evaluated 183 according to the following formula: \% Matrix effect $=100 *[($ Response of the post 184 extracted spiked sample/Response of the non-extracted neat solvent with analytes)-1]. A negative $\%$ value indicated ion suppression whereas a positive $\%$ indicated ion enhancement.

\section{Stability}

Stability of working solutions of daidzein, daidzein- $\mathrm{d}_{6}$, equol, equol- $\mathrm{d}_{4}$, and

189 genistein in methanol was evaluated to ensure that neither sample preparation nor stored 190 conditions could affect the concentration of the analytes. For this purpose, freeze and 191 thaw stability, short-term stability at room temperature, and long-term stability in the 192 freezer were evaluated. 


\section{Application to real samples: quantification of daidzein, equol and genistein} in human blood samples

\section{Study design}

A randomized, crossover, bioavailability study with a washout period of 3 days

197 was performed. The study was conducted in accordance with the Helsinki Declaration, 198 approved by the local Ethical Committee (CEIC-Parc de Salut Mar), and took place in 199 the Clinical Research Unit of Hospital del Mar Medical Research Institute (IMIM,

200 Barcelona, Spain). Written informed consent was obtained from all the participants 201 prior to any study-related procedure. Participants were financially compensated for any 202 inconvenience caused.

\section{Subjects}

204 A total of 12 healthy, non-smoker volunteers (4 female and 8 male), aged between 20 and 37 years (average $26.1 \pm 5.5$ years), were selected through a volunteer database. Their mean weight and height were $67.8 \pm 10.3 \mathrm{~kg}$ and $172.5 \pm 12.4 \mathrm{~cm}$,

207 respectively. The body mass index of each subject was within 19.6 and $25.4 \mathrm{~kg} / \mathrm{m}^{2}(22.7$

$208 \pm 1.9 \mathrm{~kg} / \mathrm{m}^{2}$ ). The women had regular menstrual cycles (26-32 days). Subjects 209 underwent a general physical examination, complete chemistry and haematology blood

210 test, urinalysis, and 12-lead ECG. Moreover, during the three days prior to the

211 intervention, they were asked to follow a diet free from daidzin, genistin, daidzein and 212 genistein (including soybeans and soy foods). A list of isoflavone rich food was 213 provided. Alcoholic beverages were not permitted 72 hours prior to each intervention.

$214 \quad$ Commercial isoflavone preparations

215 The soy isoflavone preparations used in this study were two commercially 216 available brands: Super-Absorbable Soy Isoflavones ${ }^{\circledR}$ (Life Extension, US, hard 217 gelatine capsules) and Fitoladius ${ }^{\circledR}$ (Merck, Spain, soft gelatine capsules), As provided 
218 by the manufacturer, each capsule of Super-Absorbable Soy Isoflavones ${ }^{\circledR}$ contains 123

$219 \mathrm{mg}$ of soy extract, with $54 \mathrm{mg}$ of isoflavones (44\%), $22 \mathrm{mg}$ of which are daidzin and

220 daidzein, $28 \mathrm{mg}$ genistin and genistein, and $6 \mathrm{mg}$ glycitin and glycitein. Each capsule of

221 Fitoladius ${ }^{\circledR}$ contains $60 \mathrm{mg}$ of soybean seed extract (Glycine max), with $24 \mathrm{mg}$ (40\%)

222 of total isoflavone content, the specific amount of each isoflavone is not, however,

223 described in the information leaflet.

\section{$224 \quad$ Drug administration and sample collection}

225 The treatment with Super Absorbable Soy Isoflavones ${ }^{\circledR}$ consisted of a single 226 oral intake of 2 capsules (total dose: $108 \mathrm{mg}$ isoflavones), whereas that of Fitoladius ${ }^{\circledR}$ 227 was 4 capsules (total dose: $96 \mathrm{mg}$ isoflavones). The objective of this approach was to 228 compensate the previously mentioned differences in isoflavone content of the extracts, 229 as provided by the manufacturers ( $54 \mathrm{mg}$ vs $24 \mathrm{mg}$ in each capsule). On the intervention 230 days, each volunteer received an oral dose of a soy isoflavone extract. In order to avoid 231 batch-to-batch differences in isoflavone composition, all the capsules of each treatment 232 were from the same batch number. Moreover, as explained bellow, the composition of 233 five different capsules corresponding to that batch was analysed.

234 Subjects arrived at the clinical trial unit on the intervention day at 07:45 a.m. 235 after an overnight fast. Spot urine samples were collected for drug testing (opiates, 236 cocaine, amphetamines, and cannabis) by a rapid test device (Instant-View ${ }^{\circledR}$, Alpha

237 Scientific Designs, Inc, Poway, CA, USA). In addition, a breath ethanol test was also 238 conducted. A positive screen test was considered an exclusion criterion. Intervention 239 days were separated by at least a 3-day washout period. An indwelling intravenous 240 catheter inserted into a subcutaneous vein in the forearm of the non-dominant arm and 241 blood samples were collected at baseline and $1,3,5,6,7,8,9,12$, and $24 \mathrm{~h}$ after 242 treatment administration. Blood was collected into $10 \mathrm{~mL}$ tubes containing EDTA and 
243 centrifuged (3000 rpm, $10 \mathrm{~min}, 4{ }^{\circ} \mathrm{C}$ ). The plasma samples were then separated and 244 frozen at $-20{ }^{\circ} \mathrm{C}$ until analysis.

$245 \quad$ Quantification of isoflavones in soy extract capsules

246 Although the treatment dosages (based on the information provided by the

247 manufacturers) were designed to contain similar doses of isoflavones, it was expected

248 that there would be differences in the plasmatic concentrations of specific isoflavones

249 (e.g. genistein and daidzein) as in the Fitoladius preparation their composition was not

250 declared. Consequently, it was necessary to measure the exact content of isoflavones of

251 the capsules. The isoflavone content of the preparations [aglycones (daidzein and 252 genistein) and $\beta$-glycoside conjugates (daidzin and genistin)] was determined by

253 LC/MS/MS as follows: a sample stock solution $(1 \mathrm{mg} / \mathrm{mL})$ from each was prepared by

254 dissolving $10.0 \mathrm{mg}$ of capsule content in $10 \mathrm{~mL}$ of methanol and sonicating it for 5

255 minutes to achieve complete dissolution. Analysis solutions of $100 \mu \mathrm{g} / \mathrm{mL}$ and 10

$256 \mu \mathrm{g} / \mathrm{mL}$ were prepared by further diluting the stock solution. One milliliter of the 257 analysis solution, spiked with $30 \mu \mathrm{L}$ of a solution containing daidzein- $\mathrm{d}_{6} 10 \mu \mathrm{g} / \mathrm{mL}$, was

258 subjected to the previously described solid phase extraction procedure. Ten $\mu \mathrm{L}$ of the 259 solution were injected in the LC/MS/MS system. Each determination was performed by 260 analysing five different capsules corresponding to the same batch used for the study.

261 The separation of the compounds was carried out using the same conditions described

262 earlier. The aglycones were quantified directly whereas the glycosides were quantified

263 indirectly after hydrolysis. Calculations were based on the response factor of the ratio of

264 the analyte versus internal standard.

$265 \quad$ Pharmacokinetic analysis

266 The maximum plasma concentration $\left(\mathrm{C}_{\max }\right)$ and the time to reach $\mathrm{C}_{\max }\left(\mathrm{t}_{\max }\right)$ were 267 determined from the curves constructed by plotting the plasma concentrations over 
268 time. The terminal slope $\left(\mathrm{k}_{\mathrm{e}}\right)$ of the concentration-time curve was determined by $\log$ -

269 linear regression using at least the last three points. Elimination half-life $\left(t_{1 / 2}\right)$ of the

270 terminal log-linear phase was calculated following the equation $\ln 2 / \mathrm{k}_{\mathrm{e}}$. The area under

271 the curve $\left(\mathrm{AUC}_{0-24 \mathrm{~h}}\right)$ was calculated by the trapezoidal method during the 24 hours

272 following the ingestion of the corresponding soy isoflavone extract. All the plasma

273 samples collected from each subject were analyzed in the same batch of analysis. In

274 order to compare the pharmacokinetics of genistein and daidzein in both preparations,

275 the $\mathrm{AUC}_{0-24 \mathrm{~h}}$ and $\mathrm{C}_{\max }$ were adjusted for the dose administered $\left(\mathrm{AUC}_{0-24 \mathrm{~h}} / \mathrm{Dose}\right.$ and

$276 \mathrm{C}_{\max }$ (Dose). Taking into account that the isoflavone extracts contained a mixture of

277 glycosides and aglycones, the dose administered was calculated as the sum of the

278 amount of glycoside and aglycone, expressed in mg of aglycone.

$279 \quad$ Statistical analysis

280 A Student's $t$-test for paired samples was used for the statistical comparison of

$281 \mathrm{AUC}_{0-24 \mathrm{~h}}, \mathrm{C}_{\max }, \mathrm{t}_{1 / 2}, \mathrm{k}_{\mathrm{e}}, \mathrm{AUC}_{0-24 \mathrm{~h}} /$ Dose and $\mathrm{C}_{\max } /$ Dose. A non-parametric method was

282 used (Wilcoxon signed-rank test) for $t_{\max }$. Analyses were performed using SPSS

283 Statistics for Windows (version 18.0; SPSS Inc., Chicago, IL, USA). Significance was 284 defined as $\mathrm{p}<0.05$.

285 RESULTS AND DISCUSSION

286 Method development and optimization

$287 \quad$ Optimization of MS/MS conditions

288 In order to evaluate ionization sources and multiple reaction monitoring (MRM)

289 transitions, the three most intense fragments of each compound (daidzein, daidzein- $\mathrm{d}_{6}$,

290 equol, equol- $\mathrm{d}_{4}$ and genistein) were selected using pure standards at $10 \mu \mathrm{g} / \mathrm{mL}$ in

291 negative ion mode. To further improve MRM conditions, the effect of gas temperature,

292 and collision and fragmentor energies were investigated over the range of $300-350{ }^{\circ} \mathrm{C}$, 
293 0-45 eV, and 60-140 V, respectively. The optimal gas temperature was determined at

$294325^{\circ} \mathrm{C}$, and the optimized values for fragmentor and collision energy of each

295 metabolite, as well as the selected MRM transitions, are detailed in Table 1.

296

297

298

299

300

301

302

303

304

305

306

307

308

309

310

311

312

313

314

315

316

317

\section{Optimization of LC conditions}

Different chromatographic columns were tested to study their effect on peak shapes and separation efficiency. Depending on the polarity of the stationary phase these included: cyanopropylsilyl- (CN; Acquity UPLC ${ }^{\circledR}$ HSS cyano $1.8 \mu \mathrm{m}, 2.1 \times 50$ $\mathrm{mm}), n$-octylsilyl- $\left(\mathrm{C}_{8}\right.$; Zorbax SB-C $\mathrm{C}_{8}$ RRHT $\left.1.8 \mu \mathrm{m}, 2.1 \mathrm{x} 100 \mathrm{~mm}\right)$, phenylhexylsilyl(Acquity UPLC $^{\circledR}$ BEH Phenyl $1.7 \mu \mathrm{m} 2.1 \times 100 \mathrm{~mm}$ ), and $n$-octadecylsilyl- $\left(\mathrm{C}_{18}\right.$; Acquity $\mathrm{UPLC}^{\circledR}$ BEH $\mathrm{C}_{18} 1.7 \mu \mathrm{m}, 3.0 \times 100 \mathrm{~mm}$ ) moieties on silica. After having compared the four chromatographic columns, the latter was chosen to continue with the optimization of the methodology because it provided the best separation with good resolution for equol, daidzein, and genistein.

Chromatographic separation of the analytes was optimized by evaluating four mobile phases $\left(0.1 \% \mathrm{HCOOH}, 0.01 \% \mathrm{NH}_{4} \mathrm{CH}_{3} \mathrm{COO}\right.$, acetonitrile, and methanol). $\mathrm{A}$ rapid separation $(<5 \mathrm{~min})$ of the three compounds of interest was achieved using an isocratic solvent system consisting of $0.01 \%$ ammonium acetate at $\mathrm{pH} 5$ and methanol $(40: 60, \mathrm{v} / \mathrm{v})$ (Figure 2).

\section{Optimization of the solid phase extraction procedure}

To optimize the extraction of the analytes of interest from plasma, several solidphase extraction procedures were evaluated by spiking known amounts of the analytes, and calculating the corresponding recoveries and matrix effect. Five different cartridges were evaluated. These included Oasis ${ }^{\circledR}$ MCX, WAX, MAX, WCX, and HLB (Waters corporation). The highest recovery of the analytes was achieved when MAX cartridges were used, although a marked matrix effect was present. In order to avoid the co-eluting 
318 matrix components affecting the ionization efficiency of the analytes, different washes

319 were evaluated. Neither basic washes with $5 \% \mathrm{NH}_{4} \mathrm{OH}$, nor additional washing steps

320 using methanol, improved the matrix effect. However, when acidic washes were studied

321 using mixtures of methanol and $2 \%$ formic acid, the matrix effect was enhanced. The

322 best results were obtained when $2 \% \mathrm{HCOOH}$ and methanol $(90: 10 ; \mathrm{v} / \mathrm{v})$ were

323 employed.

$324 \quad$ Optimization of the hydrolysis

325 The efficacy of different enzymes to hydrolyze the sulphate and glucuronic acid

326 moieties present in the phase II conjugates of equol, genistein, and daidzein was

327 evaluated. Different enzyme preparations ( $\beta$-Glucuronidases from Helix pomatia $\mathrm{H}-2$,

328 purified $\mathrm{H}-2, \mathrm{H}-1$, and $\beta$-Glucuronidase from Escherichia coli), glucuronidase and

329 sulphatase units, incubation times, and temperatures were studied. Several custom

330 synthesized products containing sulphate and/or glucuronide moieties (daidzein-4-

331 sulphate, equol-4-sulphate, daidzein-7- $\beta$-D-glucuronide, daidzein-7- $\beta$-D-glucuronide-4-

332 sulphate) were used as substrates and the percentage of hydrolysis achieved in each case

333 was evaluated.

334 The best results were obtained when the samples were treated with 3,000 units

335 of $\beta$-Glucuronidase $\mathrm{H}-1$ from Helix pomatia at $\mathrm{pH}=5$ after an incubation at $37^{\circ} \mathrm{C}$ for

33616 hours. Interestingly, similar results were achieved when hydrolysis time was reduced

337 to 1 hour and the temperature of the water was increased to $55^{\circ} \mathrm{C}$. This glucuronidase

338 has been previously used in the analysis of soy isoflavones ${ }^{18-21}$.

339 Under the conditions described above, the percentage of hydrolysis of daidzein-

340 4-sulphate, daidzein-7- $\beta$-D-glucuronide, and daidzein-7- $\beta$-D-glucuronide-4-sulphate

341 was higher than $95 \%$. With greater amounts of $\beta$-glucuronidase (5,000 units), the

342 percentage of hydrolysis was constant although the matrix effect increased. Whilst the 
343 hydrolysis of most of the metabolites was successful, none of the studied conditions was

344 able to hydrolyze equol-4-sulphate, the yield being below 5\%. Studies regarding equol 345 metabolism in humans, monkeys, and rats have reported that sulphation is a minor

346 metabolic pathway of this compound. ${ }^{22}$ As described below, despite having detected

347 equol in plasma samples from three volunteers, the pharmacokinetics of this metabolite 348 has not been evaluated in this report.

349 It is worth noting that a total quantification of isoflavones was chosen due to the

350 large number of isoflavone glucuronide and sulphate metabolites conjugates that have

351 been described to date in humans ${ }^{16}$ and animals. ${ }^{9}$ Although some of these conjugates are 352 commercially available, the lack of an appropriate commercially available IS of all of 353 them for their proper quantification was a major limitation for their inclusion in the 354 bioavailability study. For this reason, a total quantification of the isoflavones was 355 performed following sample hydrolysis. To do so, four different representative 356 metabolites presenting glucuronides and/or sulphate moieties were custom synthesized.

357 The hydrolysis procedure was optimized in order to assure that the conditions used were 358 able to release the aglycones. Finally, the quantification of total isoflavones was 359 performed in real plasma samples using the optimized hydrolysis procedure

$360 \quad$ Method validation

361 Validation of the analytical method was performed following the European 362 Medicines Agency guidelines on bioanalytical method validation (URL 363 http://www.ema.europa.eu/docs/en_GB/document_library/

364 Scientific_guideline/2011/08/WC500109686.pdf . Accessed 30.04.2014).

365 The linearity of the calibration curve was evaluated from three different,

366 prepared batches as described above. Satisfactory determination coefficients $\left(r^{2}>0.99\right)$

367 were obtained for all the analytes in the selected range of 5-1000 ng/mL. 
Intra- and inter-day accuracies presented acceptable \% relative error for the three compounds, and the intra- and inter-day precision were constantly below $15 \%$ RSD

370

371

\section{(Supplementary Table 1).}

The LOD for daidzein, equol, and genistein were $0.6,0.9$, and $1.0 \mathrm{ng} / \mathrm{mL}$, respectively. The LLOQ for these three isoflavones was $5 \mathrm{ng} / \mathrm{mL}$.

The $\%$ matrix effect for daidzein, equol, genistein, and their corresponding IS assessed at 5 and $800 \mathrm{ng} / \mathrm{mL}$, indicate that ion suppression was lower than $17.2 \%$ and ion enhancement was not higher than $17.8 \%$. The extraction recoveries were between 77.5 and $86 \%$ (Table 2).

No significant degradation of daidzein, equol, and genistein in human plasma took place after long-term storage at $-20{ }^{\circ} \mathrm{C}$ during the analysis period. The low and high QC samples presented acceptable values ( $\pm 15 \%$ the nominal values) during at least 8 months after their preparation.

\section{Quantification of isoflavones in soy preparations}

Supplementary Table 2 shows the quantification of daidzein, genistein, daidzin, and genistin present in one capsule of the commercially available soy preparations studied. Both commercial sources presented predominantly $\beta$-glycoside conjugates (98\%), the free forms representing only a small percentage of the isoflavone content (approximately $2 \%$ ). In Fitoladius ${ }^{\circledR}$, daidzin was found to account for $37.4 \%$ of the total isoflavone weight, whereas genistin was the most abundant isoflavone, representing $60.3 \%$. On the other hand, Super Absorbable Soy Isoflavones ${ }^{\circledR}$ had almost the same amount of daidzin as genistin $(50.0 \%$ and $48.1 \%$, respectively). The aglycones were only present in very low amounts, being daidzein at a higher proportion (1.1-1.7\%) than genistein $(0.6-0.7 \%)$. The total isoflavone content of Fitoladius ${ }^{\circledR}$ was found to exactly match the content provided in the information pamphlet (23.8 $\mathrm{mg}$ vs $24 \mathrm{mg}$ ). In the case 
393 of Soy Isoflavones ${ }^{\circledR}$, the content of genistin and genistein was 1.2-fold that described

394 by the manufacturer ( $35.5 \mathrm{mg}$ vs $28 \mathrm{mg}$ ). As for daidzein and daidzin concerns, the

395 amount quantified (34.5 mg) was 1.6-fold that given in the specifications (22 $\mathrm{mg})$. This

396 fact is of relevance, since the dosages administered to the volunteers were based on the

397 information provided by the manufacturer. In order to compensate for these differences,

398 the pharmacokinetic parameters were normalised by the dose administered. To do so,

399 each plasmatic concentration value (derived from the calibration curve) was divided by

400 the amount administered of the corresponding isoflavone.

401 The other constituents present in the soy preparations were excipients. In the case of

402 Fitoladius ${ }^{\circledR}$, and according to the manufacturer, these are primrose oil, glyceryl

403 monostearate, glycerol, cuprosodic chlorophyll, gelatine, titanium dioxide and purified

404 water. In the case of Super-Absorbable Soy Isoflavones ${ }^{\circledR}$, these are ascorbyl palmitate

405 and vegetable cellulose. The analysis of these excipients is beyond the scope of this 406 paper.

$407 \quad$ Quantification of isoflavones in human plasma samples

$408 \quad$ Equol

409 Only three of the twelve volunteers were found to be equol producers. In these

410 volunteers, equol concentrations started to increase between 5 and 6 hours after the

411 treatment administration and could still be detected at $24 \mathrm{~h}$. Due to this fact, the

412 pharmacokinetics of this metabolite is not evaluated in this report.

$413 \quad$ Daidzein and genistein pharmacokinetics

414 Time-course plasma concentrations of total genistein and daidzein are shown in

415 Figure 3. Some studies have described that daidzein presents higher bioavailability than

416 genistein ${ }^{23}$ whereas others have reported the contrary. ${ }^{24}$ In our study, no differences in

417 bioavailability were observed between daidzein and genistein after the treatment with 
418 Super Absorbable Soy Isoflavones ${ }^{\circledR}$ or Fitoladius ${ }^{\circledR}$. The double peak found in the time-

419 course plasma concentrations suggests an enterohepatic circulation, which is

420 characteristic of these compounds (Figure 3). ${ }^{6,25}$ To a lesser extent, the small

421 percentages of aglycones present in the soy extracts could also contribute to this

422 pharmacokinetic profile, as the free forms are absorbed faster than the glycosides. ${ }^{26}$

423 The pharmacokinetic parameters studied $\left(\mathrm{AUC}_{0-24 \mathrm{~h}}, \mathrm{C}_{\max }, \mathrm{AUC}_{0-24 \mathrm{~h}} / \mathrm{Dose}\right.$,

$424 \mathrm{C}_{\max } /$ Dose, $\mathrm{t}_{\max }, \mathrm{t}_{1 / 2}, \mathrm{k}_{\mathrm{e}}$ ) are reported in Table 3. As shown using the $\mathrm{AUC}_{0-24 \mathrm{~h}} /$ Dose

425 ratio, the bioavailability of daidzein and genistein was higher after the intake of

426 Fitoladius ${ }^{\circledR}(\mathrm{p}<0.05$ and $\mathrm{p}<0.001$, respectively), compared to Super Absorbable Soy

427 Isoflavones ${ }^{\circledR}$ (Figure 3).

428 Cmax/Dose values of genistein also differed significantly between treatments $429(\mathrm{p}<0.001)$. After the intake of Fitoladius ${ }^{\circledR}$ these values were higher than the other 430 studied preparation. No differences were observed in $t_{\max }, t_{1 / 2}, k_{\mathrm{e}}$.

431 Several factors can influence the metabolism and bioavailability of isoflavones in 432 humans. These include age, ethnic background, dietary habits, food matrix, intestinal 433 microflora, intestinal transit time, previous exposure to isoflavones, and glycosidase 434 production. $^{27,} 28$ In our case, the differences in bioavailability suggest that the 435 excipients/matrix in which these products are present may play an important role in the 436 absorption process, which could have a direct impact on the bioavailability of soy 437 isoflavones. This fact would explain the different pharmacokinetic profile observed 438 between treatments when the same 12 volunteers received two different soy extracts.

439 Our study has strengths and limitations. One of the strengths is the crossover design, 440 which allowed the same participants to be included in the different treatments, thus, 441 minimising interferences with other confounder variables. Another strength lies in the 442 fact of including both genders among the participants, thus, having a better 
443 representation of the overall population. One limitation of our study is that, due to the

444 available commercial sources of the soy extracts, the isoflavone composition of the two

445 tested products differed significantly. Consequently, the participants did not receive

446 exactly the same doses in the two soy isoflavone extracts. In order to minimise these

447 differences, the exact amount of each isoflavone was firstly analysed, and normalisation

448 by the dose administered was performed prior to pharmacokinetic analyses.

449 In conclusion, in the present study we developed a LC/MS/MS method based on an

450 enzymatic hydrolysis with $\beta$-glucuronidase $\mathrm{H} 1$ followed by SPE procedure using

451 Oasis ${ }^{\circledR}$ MAX cartridges. The analytical method was successfully validated following

452 the European Medicines Agency guidelines on Bioanalytical Method Validation. This

453 methodology presents a good recovery of the analytes, very low matrix effects, a rapid

454 chromatographic separation and high sensitivity. The limits of detection for daidzein,

455 equol, and genistein in $1 \mathrm{~mL}$ of plasma sample were $0.6,0.9$ and $1.0 \mathrm{ng} / \mathrm{mL}$,

456 respectively. Once the method was validated, a crossover, randomized, bioavailability

457 study was performed. The method was then applied to the plasma samples of the 12

458 volunteers. The pharmacokinetic profile of daidzein and genistein was studied.

459 After having been normalized, Fitoladius ${ }^{\circledR}$ presented higher bioavailability and

460 achieved higher Cmax values than Super Absorbable Soy Isoflavones ${ }^{\circledR}$ for daidzein and

461 genistein. As the other pharmacokinetic parameters were similar between both

462 treatments, we suggest that these differences in bioavailability come from the

463 matrix/excipients present in the capsules of the isoflavone extracts.

464 The preparations studied are over-the-counter dietetic preparations, they do not 465 require medical prescription, and some of them can be purchased on the internet. Thus, 466 the regulation of these products is not as strict as that applied to prescription drugs.

467 Consequently, a detailed and accurate description of the specific isoflavone content 
468 present in the capsules is lacking. Indeed, describing the content of the capsules as " $\mathrm{X}$ $469 \mathrm{mg}$ of isoflavones" or "Y mg of genistin and genistein" does not provide clear and 470 accurate information about composition. This is an important fact since there are a 471 number of different isoflavones, which vary in molecular weight, pharmacokinetic 472 profile, and biological activities. ${ }^{29}$ As a consequence, the exact content of each 473 isoflavone should be provided by the manufacturers in order to have a better knowledge 474 of the content of the capsules and their corresponding beneficial health effects.

475 The purpose of this study was the selection of one isoflavone extract preparation for 476 its use in a clinical trial with the aim of treating drug addiction. In this context we 477 performed the bioavailability study. Results from this report not only show that 478 isoflavones extracts vary in their composition (not unexpected) but also in the amounts 479 reported by manufacturers in each preparation. In a clinical pharmacology context and 480 looking at therapeutic indications with methodological robust designs, results from this 481 study are quite disturbing.

482 


\section{Supporting Information:}

484 Supplementary Table 1. Intra- and inter-day precision and accuracy of quality 485 control samples of daidzein, equol and genistein in human plasma.

486

487 Supplementary Table 2. Isoflavone content of one capsule of Fitoladius ${ }^{\circledR}$ and 488 Super Absorbable Soy Isoflavones ${ }^{\circledR}$. Data are expressed in milligrams as mean \pm 489 standard deviation.

490

491 This material is available free of charge via the Internet at http://pubs.acs.org

492 


\section{REFERENCES}

494 1. Yuan, J. P.; Wang, J. H.; Liu, X., Metabolism of dietary soy isoflavones to equol by 495 human intestinal microflora--implications for health. Mol. Nutr. Food Res. 2007, 51, $496 \quad 765-81$.

497 2. Milenkovic, D.; Jude, B.; Morand, C., miRNA as molecular target of polyphenols 498 underlying their biological effects. Free Radical Bio. Med. 2013, 64, 40-51.

499 3. Day, A. J.; DuPont, M. S.; Ridley, S.; Rhodes, M.; Rhodes, M. J.; Morgan, M. R.;

500 Williamson, G., Deglycosylation of flavonoid and isoflavonoid glycosides by human 501 small intestine and liver beta-glucosidase activity. EEBS Letters 1998, 436, 71-5.

502 4. Setchell, K. D.; Brown, N. M.; Zimmer-Nechemias, L.; Brashear, W. T.; Wolfe, B. 503 E.; Kirschner, A. S.; Heubi, J. E., Evidence for lack of absorption of soy isoflavone 504 glycosides in humans, supporting the crucial role of intestinal metabolism for 505 bioavailability. Am. J. Clin. Nutr. 2002, 76, 447-53.

506 5. Kurzer, M. S.; Xu, X., Dietary phytoestrogens. Annu. Rev. Nut. 1997, 17, 353-81.

507 6. Barnes, S., The biochemistry, chemistry and physiology of the isoflavones in 508 soybeans and their food products. Lumphat.Res.Biol. 2010, 8, 89-98.

509 7. Ronis, M. J.; Little, J. M.; Barone, G. W.; Chen, G.; Radominska-Pandya, A.;

510 Badger, T. M., Sulfation of the isoflavones genistein and daidzein in human and rat

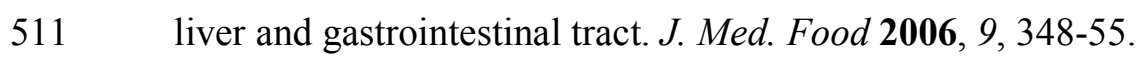

512 8. Nakano, H.; Ogura, K.; Takahashi, E.; Harada, T.; Nishiyama, T.; Muro, K.; 513 Hiratsuka, A.; Kadota, S.; Watabe, T., Regioselective monosulfation and disulfation 514 of the phytoestrogens daidzein and genistein by human liver sulfotransferases. Drug $515 \quad$ Metab. Pharmacok. 2004, 19, 216-26. 
516 9. Fang, N.; Yu, S.; Badger, T. M., Characterization of Isoflavones and Their

517 Conjugates in Female Rat Urine Using LC/MS/MS. J. Agr. Food Chem. 2002, 50, $518 \quad 2700-2707$.

519 10. Yuan, B.; Zhen, H.; Jin, Y.; Xu, L.; Jiang, X.; Sun, S.; Li, C.; Xu, H., Absorption 520 and plasma disposition of genistin differ from those of genistein in healthy women. $J$. 521 Agr. Food Chem. 2012, 60, 1428-36.

522 11. Pugazhendhi, D.; Watson, K. A.; Mills, S.; Botting, N.; Pope, G. S.; Darbre, P. 523 D., Effect of sulphation on the oestrogen agonist activity of the phytoestrogens 524 genistein and daidzein in MCF-7 human breast cancer cells. J. Endocrinol. 2008, $525 \quad 197,503-515$.

526 12. Xu, X.; Harris, K. S.; Wang, H. J.; Murphy, P. A.; Hendrich, S., Bioavailability 527 of soybean isoflavones depends upon gut microflora in women. J. Nutr. 1995, 125, $528 \quad 2307-15$.

529 13. Setchell, K. D.; Clerici, C.; Lephart, E. D.; Cole, S. J.; Heenan, C.; Castellani, $530 \quad$ D.; Wolfe, B. E.; Nechemias-Zimmer, L.; Brown, N. M.; Lund, T. D.; Handa, R. J.; 531 Heubi, J. E., S-equol, a potent ligand for estrogen receptor beta, is the exclusive 532 enantiomeric form of the soy isoflavone metabolite produced by human intestinal 533 bacterial flora. Am. J. Clin. Nutr. 2005, 81, 1072-9.

534 14. Setchell, K. D.; Brown, N. M.; Desai, P.; Zimmer-Nechemias, L.; Wolfe, B. E.; 535 Brashear, W. T.; Kirschner, A. S.; Cassidy, A.; Heubi, J. E., Bioavailability of pure 536 isoflavones in healthy humans and analysis of commercial soy isoflavone 537 supplements. J. Nutr. 2001, 131, 1362S-75S.

538 15. King, R. A.; Bursill, D. B., Plasma and urinary kinetics of the isoflavones 539 daidzein and genistein after a single soy meal in humans. Am. J. Clin. Nutr. 1998, 67, $540 \quad 867-72$ 
541 16. Hosoda, K.; Furuta, T.; Ishii, K., Simultaneous determination of glucuronic acid 542 and sulfuric acid conjugated metabolites of daidzein and genistein in human plasma

543 by high-performance liquid chromatography. J.Chromatogr. B 2010, 878, 628-36.

544 17. Twaddle, N. C.; Churchwell, M. I.; Doerge, D. R., High-throughput 545 quantification of soy isoflavones in human and rodent blood using liquid 546 chromatography with electrospray mass spectrometry and tandem mass spectrometry 547 detection. J. Chromatogr. B 2002, 777, 139-45.

548 18. Wyns, C.; Bolca, S.; De Keukeleire, D.; Heyerick, A., Development of a high549 throughput LC/APCI-MS method for the determination of thirteen phytoestrogens 550 including gut microbial metabolites in human urine and serum. J. Chromatogr. B $551 \quad 2010,878,949-956$.

552 19. Kuklenyik, Z.; Ye, X.; Reich, J. A.; Needham, L. L.; Calafat, A. M., Automated 553 online and off-line solid-phase extraction methods for measuring isoflavones and 554 lignans in urine. J. Chromatogr. Sci. 2004, 42, 495-500.

555 20. Parker, D. L.; Rybak, M. E.; Pfeiffer, C. M., Phytoestrogen biomonitoring: an 556 extractionless LC-MS/MS method for measuring urinary isoflavones and lignans by 557 use of atmospheric pressure photoionization (APPI). Anal. Bioanal. Chem. 2012, $558 \quad 402,1123-36$.

559 21. Rybak, M. E.; Parker, D. L.; Pfeiffer, C. M., Determination of urinary 560 phytoestrogens by HPLC-MS/MS: A comparison of atmospheric pressure chemical 561 ionization (APCI) and electrospray ionization (ESI). J. Chromatogr. B 2008, 861, $562 \quad 145-150$.

563 22. Schwen, R. J.; Nguyen, L.; Jackson, R. L., Elucidation of the metabolic pathway 564 of S-equol in rat, monkey and man. Food. Chem. Toxicol. 2012, 50, 2074-83. 
565 23. Xu, X.; Wang, H.-J.; Murphy, P. A.; Cook, L.; Hendrich, S., Daidzein Is a More

566 Bioavailable Soymilk Isoflavone than Is Genistein in Adult Women. J. Nutr. 1994, $567 \quad 124,825-832$.

568 24. Setchell, K. D.; Faughnan, M. S.; Avades, T.; Zimmer-Nechemias, L.; Brown, 569 N. M.; Wolfe, B. E.; Brashear, W. T.; Desai, P.; Oldfield, M. F.; Botting, N. P.; 570 Cassidy, A., Comparing the pharmacokinetics of daidzein and genistein with the use 571 of ${ }^{13} \mathrm{C}$-labeled tracers in premenopausal women. Am. J. Clin. Nutr. 2003, 77, 411-9.

572 25. Sfakianos, J.; Coward, L.; Kirk, M.; Barnes, S., Intestinal uptake and biliary 573 excretion of the isoflavone genistein in rats. J.Nutr. 1997, 127, 1260-8.

574 26. Izumi, T.; Piskula, M. K.; Osawa, S.; Obata, A.; Tobe, K.; Saito, M.; Kataoka, 575 S.; Kubota, Y.; Kikuchi, M., Soy isoflavone aglycones are absorbed faster and in 576 higher amounts than their glucosides in humans. J.Nutr. 2000, 130, 1695-9.

577 27. Zubik, L.; Meydani, M., Bioavailability of soybean isoflavones from aglycone 578 and glucoside forms in American women. Am. J. Clin. Nutr. 2003, 77, 1459-65.

579 28. Setchell, K. D., Phytoestrogens: the biochemistry, physiology, and implications 580 for human health of soy isoflavones. Am.J.Clin. Nutr. 1998, 68, 1333S-1346S.

581 29. López-Gutiérrez, N.; Romero-González, R.; Garrido Frenich, A.; Martínez 582 Vidal, J. L., Identification and quantification of the main isoflavones and other 583 phytochemicals in soy based nutraceutical products by liquid chromatography584 orbitrap high resolution mass spectrometry. LChromatogr.A 2014, 1348, 125-136.

Note of acknowledgement for funding: Funded by grants from the Ministerio de Sanidad (Investigación Clínica Independiente, EC-10-166), Fondo de Investigación Sanitaria-ISCIII-FEDER RTA (RD12/0028/0009), CIBEROBN (CB06/03/0028), Generalitat de Catalunya (AGAUR 2014SGR680, predoctoral fellowship FI-DGR 2013 
for JRM) and PN I+D+I 2008-2011 and ISCIII CA11/00215 for NP. CPM and EP are Rio Hortega fellows (ISC-III, CM12/00085 and CM13/00016). The author's work was independent of the sources of funding. 


\section{Figure captions}

Figure 1. Chemical structures of daidzein, equol and genistein.

Figure 2. Chromatogram of an extracted plasma sample. A blank plasma was spiked with daidzein, equol and genistein $(500 \mathrm{ng} / \mathrm{mL})$, and their corresponding internal standards (300 and $100 \mathrm{ng} / \mathrm{mL}$ ). Following extraction and using an isocratic gradient, analytes were separated within 5 minutes.

Figure 3. Time-course plasma concentrations of genistein and daidzein after a single oral administration of two different isoflavone extracts to human subjects $(n=12)$. For comparison purposes, the concentration values were normalized by the dose administered. A, Daidzein time-course plasmatic concentrations after Super Absorbable Soy Isoflavones ${ }^{\circledR}$ and Fitoladius ${ }^{\circledR}$ treatment; B, Genistein time-course plasmatic concentrations after treatments. Data are expressed as mean \pm standard error of the mean. 


\section{Tables}

Table 1. MS/MS Condition Settings

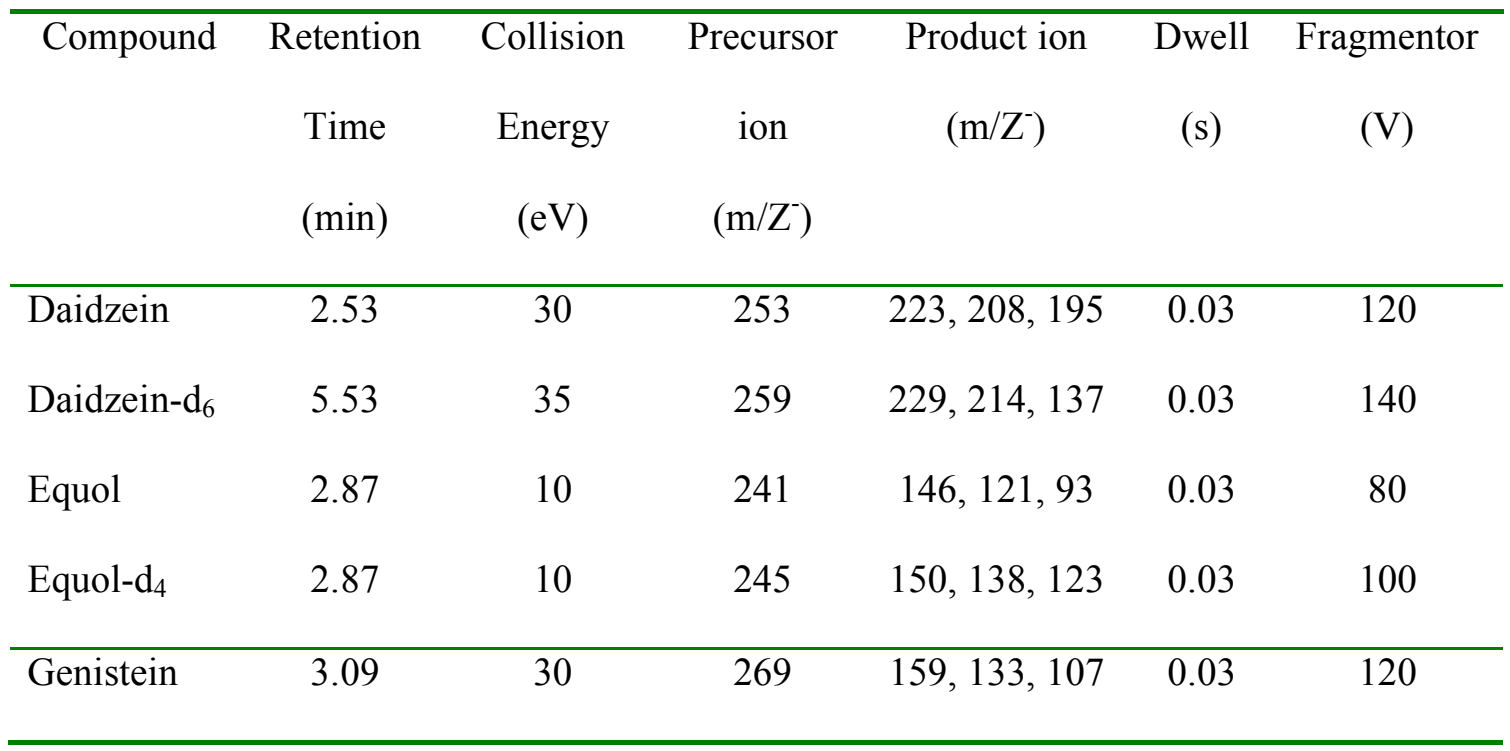


Table 2. Linearity, Limit of Detection, Matrix Effect and Extraction Recovery for the Studied Analytes.

\begin{tabular}{lcccccc}
\hline Compound & Determination & Limit of & \multicolumn{2}{c}{ Matrix effect (\%) } & \multicolumn{2}{c}{ Extraction recovery (\%) } \\
\cline { 4 - 6 } & coefficient & detection & $5 \mathrm{ng} / \mathrm{mL}$ & $800 \mathrm{ng} / \mathrm{mL}$ & $5 \mathrm{ng} / \mathrm{mL}$ & $800 \mathrm{ng} / \mathrm{mL}$ \\
& $\left(\mathrm{r}^{2}\right)$ & $(\mathrm{ng} / \mathrm{mL})$ & & & & \\
Daidzein & 0.999 & 0.6 & 17.8 & 2.1 & 86.2 & 85.9 \\
Daidzein-d & - & - & -7.1 & -11.6 & 79.2 & 77.5 \\
Equol & 0.999 & 0.9 & -7.6 & -4.8 & 79.0 & 84.5 \\
Equol-d & - & - & -12.3 & -17.2 & 76.7 & 80.2 \\
\hline Genistein & 0.996 & 1.0 & -1.8 & -9.4 & 79.1 & 80.5 \\
\hline
\end{tabular}


Table 3. Plasma Pharmacokinetic Parameters of Daidzein and Genistein after a Single Oral Administration of Fitoladius ${ }^{\circledR}$ ( 4 capsules) and Super Absorbable Soy Isoflavones ${ }^{\circledR}$ ( 2 capsules). Data Are Expressed as Mean \pm Standard Deviation.

\begin{tabular}{|l|c|c|c|c|}
\hline & \multicolumn{2}{|c|}{ Daidzein } & \multicolumn{2}{c|}{ Genistein } \\
\hline & Soy Isoflavones ${ }^{\circledR}$ & Fitoladius ${ }^{\circledR}$ & Soy Isoflavones ${ }^{\circledR}$ & Fitoladius ${ }^{\circledR}$ \\
\hline $\mathrm{AUC}_{0-24 \mathrm{~h}}{ }^{a}(\mathrm{ng} \cdot \mathrm{h} / \mathrm{mL})$ & $5408 \pm 928^{* *}$ & $3376 \pm 839^{* *}$ & $5723 \pm 1587$ & $5838 \pm 1275$ \\
\hline $\begin{array}{l}\mathrm{AUC}_{0-24 \mathrm{~h}} / \mathrm{Dose} \\
(\mathrm{ng} \cdot \mathrm{h} / \mathrm{mL} \cdot \mathrm{mg})\end{array}$ & $120.8 \pm 20.7^{*}$ & $144.8 \pm 36.0^{*}$ & $131.5 \pm 36.5^{* *}$ & $159.7 \pm 34.9^{* *}$ \\
\hline $\mathrm{C}_{\max }{ }^{b}(\mathrm{ng} / \mathrm{mL})$ & $618.6 \pm 116.8^{* *}$ & $350.3 \pm 90.8^{* *}$ & $630.8 \pm 206.3$ & $620.5 \pm 196.9$ \\
\hline $\mathrm{C}_{\max } / \mathrm{Dose}(\mathrm{ng} / \mathrm{mL} \cdot \mathrm{mg})$ & $13.8 \pm 2.6$ & $15.0 \pm 3.9$ & $14.5 \pm 4.7^{*}$ & $17.0 \pm 5.4^{*}$ \\
\hline $\mathrm{t}_{\max }{ }^{c}(\mathrm{~h})$ & $6.3 \pm 2.0$ & $6.8 \pm 0.8$ & $6.3 \pm 2.1$ & $6.9 \pm 1.0$ \\
\hline $\mathrm{t}_{1 / 2}{ }^{d}(\mathrm{~h})$ & $4.5 \pm 1.6$ & $5.4 \pm 3.3$ & $5.9 \pm 2.1$ & $5.8 \pm 2.6$ \\
\hline $\mathrm{k}_{\mathrm{e}}{ }^{e}\left(\mathrm{~h}^{-1}\right)$ & $0.18 \pm 0.07$ & $0.16 \pm 0.07$ & $0.13 \pm 0.05$ & $0.14 \pm 0.07$ \\
\hline
\end{tabular}

Abbreviations: ${ }^{a} \mathrm{AUC}_{0-24 \mathrm{~h}}$ : Area under the curve from 0 to $24 \mathrm{~h} ;{ }^{b} \mathrm{C}_{\max }$ : Peak plasma concentration; ${ }^{c} \mathrm{t}_{\max }$ : Time at peak plasma concentration; ${ }^{d} \mathrm{t}_{1 / 2}$ : elimination half-life; ${ }^{e} \mathrm{k}_{\mathrm{e}}$ : elimination rate constant. ${ }^{*} p<0.05 ; * * p<0.005$ 
Figure 1.

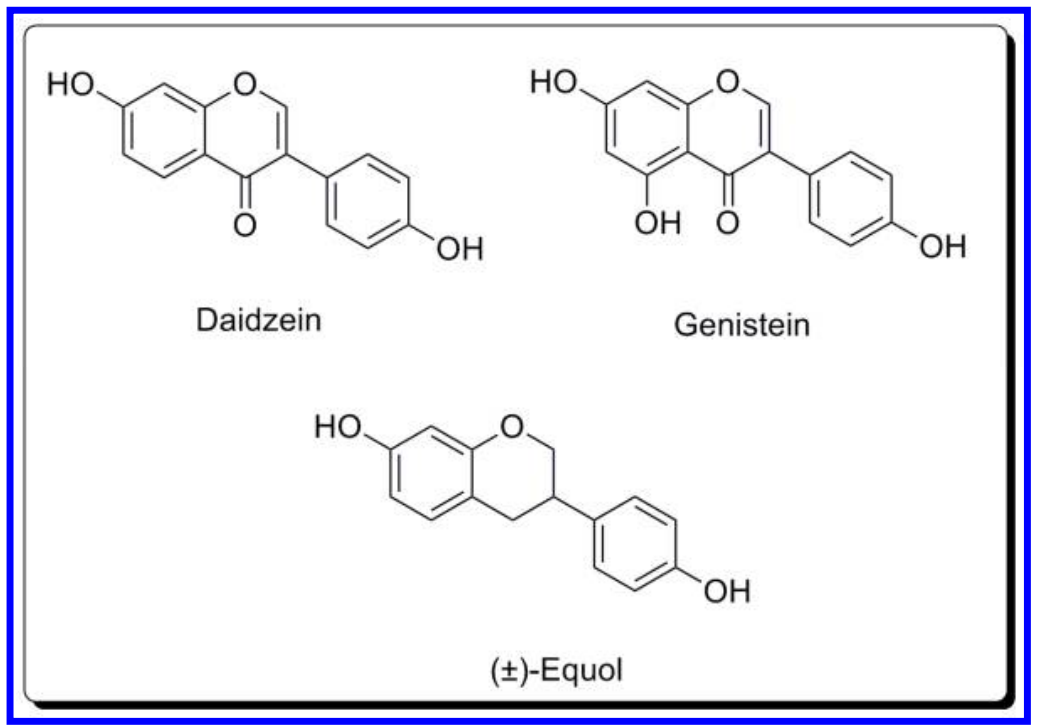


Figure 2.

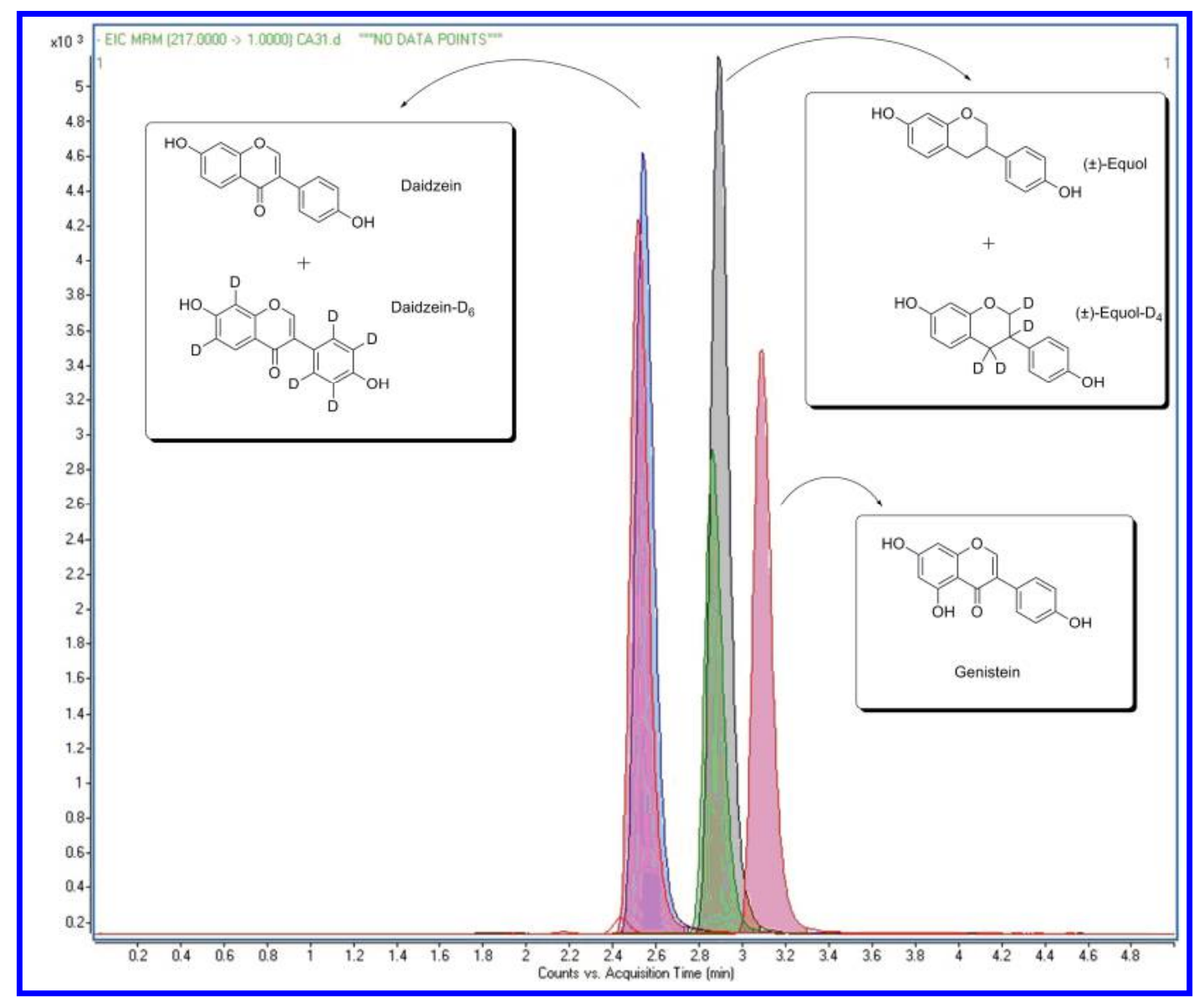


Figure 3.

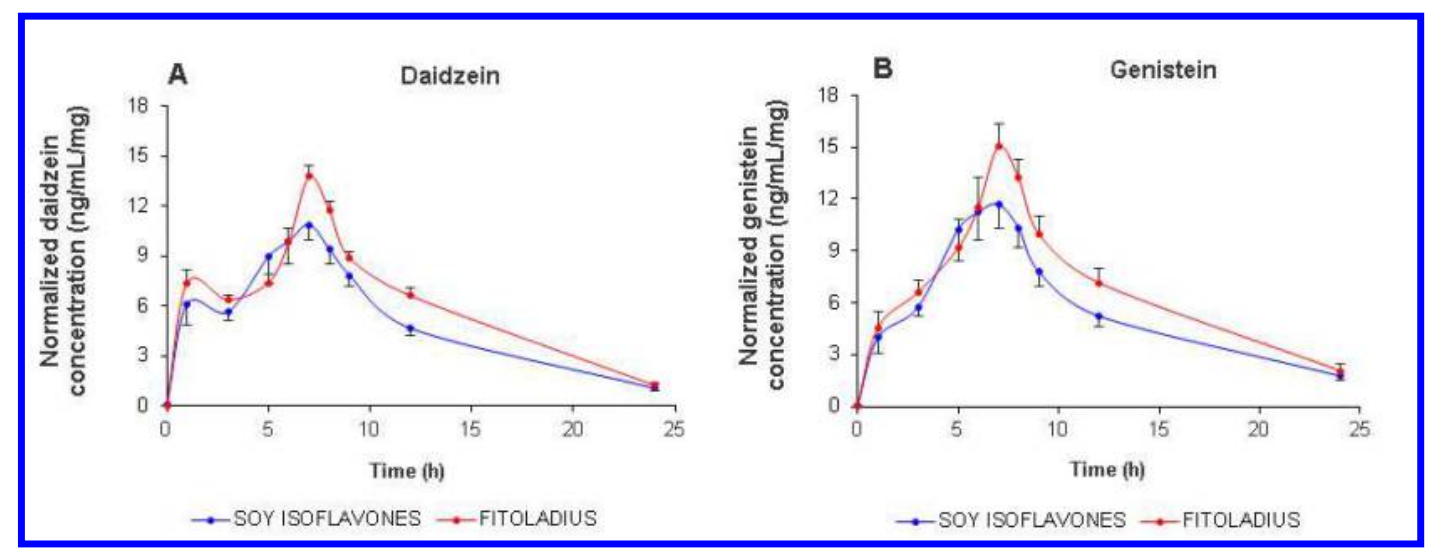




\section{TOC Graphic}

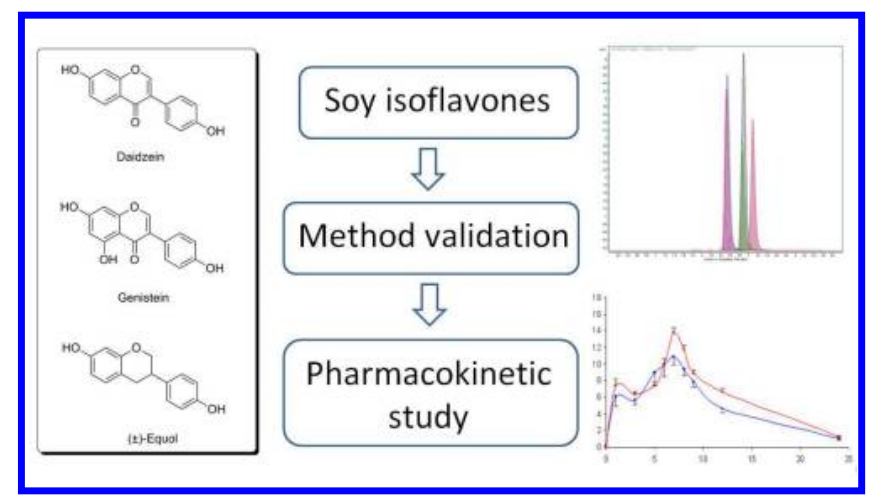

